\title{
Amino acid diversity on the basis of cytochrome b gene in Kacang and Ettawa Grade goats
}

\author{
D. A. Lestari, Sutopo and E. Kurnianto \\ Faculty of Animal and Agricultural Sciences, Diponegoro University, \\ Tembalang Campus, Semarang 50275 - Indonesia. \\ Corresponding E-mail : kurniantoedy17@gmail.com
}

Received April 28, 2017; Accepted June 03, 2017

\begin{abstract}
ABSTRAK
Tujuan penelitian adalah untuk mengidentifikasi dan mengkaji keragaman asam amino gen Cytochrome B (Cyt b), penanda genetik dan karakteristik asam amino spesifik pada kambing Kacang dan Kambing Peranakan Ettawa (PE).Materi penelitian menggunakan 19 ekor kambing Kacang dan 12 ekor kambing PE. Sample ditentukan dengan purposive sampling. DNA total diisolasi menggunakan kit Genomic DNA Mini Kit (Geneaid) dan gen Cyt b diamplifikasi dengan metode PCR menggunakan primer CytbCapF dan CytbCapR dan dilakukan sekuensing. Hasil menunjukkan terdapat 2 asam amino spesifik yang membedakan kambing Kacang dan PE dengan C. hircus dan C. aegagrus dan 4 asam amino spesifik yang membedakan dengan $C$. falconeri, namun tidak ada asam amino spesifik yang dapat membedakan kambing Kacang dengan PE. Dapat disimpulkan bahwa asam amino spesifik pada gen Cyt $\mathrm{b}$ dapat digunakan sebagai penanda genetik yang membedakan antara kambing Kacang dan PE dengan 3 kambing pembanding lainnya.
\end{abstract}

Kata-kata kunci: asam amino, cytochrome b, kambing lokal, penanda genetik

\section{ABSTRACT}

The objectives of study were to identify and assess the amino acid diversity of Cytochrome b (Cyt b) gene, genetic marker and characteristic of specific amino acid in Kacang and Ettawa Grade goat. Nineteen heads of Kacang goat (KG) and twelve heads of Ettawa Grade goat (EG) were purposively sampled. The genomic DNA was isolated by Genomic DNA Mini Kit (Geneaid) and amplified Cyt b using PCR method with CytbCapF and CytbCapR primers and was sequenced. The results showed that there were two specific amino acids that distinguish $\mathrm{KG}$ and $\mathrm{EG}$ goat with $C$. hircus and C. aegagrus and four specific amino acids that distinguish KG and EG goat with $C$. falconeri, but there were no specific amino acids can be used as a genetic marker to distinguish between Kacang and EG goat. In conclusion, specific amino acids in Cyt b gene can be used as a genetic marker among KG and EG goat with 3 goat others comparator.

Keywords : amino acid, cytochrome b, genetic marker, local goat

\section{INTRODUCTION}

Indonesia has a high diversity of local animal genetic resources. Local goats constitute an important genetic resources of the country. The Ministry of Agriculture (2016) reported that total goat population in Indonesia in 2015 was about more than 19 milion heads, the highest population in Central Java province as many as about more than 4 million heads, or $21,40 \%$ of the total population in Indonesia. Sodiq and Zainal (2008) stated that Central Java is the province with the largest population of goats. Kacang and Ettawa Grade (EG) goats are meat type goats that occur widely in the Central Java province. Kacang goat is an Indonesian native goat which are scattered in 
various regions in Indonesia (Prawirodigdo et al., 2003). While EG goat is result of cross between Kacang and Ettawa breeds, and it looks similar to Ettawa but smaller and has two or three coat color patterns, namely black striped, brown striped, mottled white and black (Batubara et al., 2009).

Understanding the genetic diversity of a population is very important conservation and breeding program. For conservation, it is necessary to know the genetic status of a population in order to design a conservation program to avoid extinction and help to develop management plans for animal survival. Genetic diversity reflects the genetic resources needed for ecological adaptation in evolution (Poerba and Yuzammi, 2008). High genetic diversity will greatly help a population to adapt to changes that occur in their surroundings and have a high breeding rate. Also genetic diversity information is required in selection during animal breeding (Santoso et al., 2006). Long-term breeding programs that utilize germplasm to improve traits of a livestock should be based on the accurate estimation of genetic determination, therefore determination of an individual as material can be done properly in genetic improvement (Karsinah et al., 2002).

Genetic diversity information can be determined in two ways, through phenotype and genotype traits. Through genotype traits, it can be done by mitochondrial DNA (mtDNA) analysis. It is one of many methods that is used to study the origin of domesticated animal (Machugh and Bradley, 2001). Cytochrome B gene (Cyt b) that contained in mitochondrial DNA, is a gene that is involved in the transport of electrons in the formation of energy respiratory chain (Anderson et al., 1981). Cyt b gene is inherited maternaly and used widely for research or phylogeny identification and genetic relationship among the species of the same genus or family. It is because Cyt $b$ gene is one of coding regions for protein in mitochondrial genome and the region is conserve or do not change much or do not experience bases mutation, thus it will be more sensitive to be used as a genetic marker for identifying the purity of the species (Manceau et al.,1999; Widayanti et al., 2006). Research on using Cyt b gene as genetic marker for genetic information analysis on goat was done in Chinese goat (Chen et al., 2006; Liu et al., 2009), Vietnamese goat (Hassanin et al., 2010), Turkish goat (Kul and Ertugrul, 2011) and Indonesian goat (Jiyanto et al., 2014; Pakpahan et al., 2016a; Pakpahan et al., 2016b).
The objective of this study was to identify and assess the amino acid diversity of Cyt $b$ gene, genetic marker and characteristic of specific amino acid in Kacang and EG goat.

\section{MATERIALS AND METHODS}

\section{Sample Collection}

Materials of this research were 31 DNA goats from blood that were taken from vena jugularis. These were obtained from two breeds of local goat, which consisted of 19 heads of Kacang goat $(\mathrm{KG})$ from Grobogan regency and 12 heads of Ettawa Grade goat (EG) from Kendal regency. Sample was determined using purposive sampling, based on criteria that the research location were selected based on regions with the largest population and development area of Kacang goat and EG goat; the goat must be 1-2 years old and did not have a genetic relationship between one sample to other samples.

\section{DNA Extraction, PCR Amplification and Sequencing}

Total genomic DNA was extracted from blood by using Genomic DNA Mini Kit (Geneaid) with Sambrook et al. (1989) method with modification following the protocol. The results of DNA extraction were visualized using $1 \%$ gel Agarose electrophoresis. Electrophoresis was run on $100 \mathrm{~V}$ condition for $45 \mathrm{~min}$ and whole genome DNA result could be seen on UV light.

The complete mitochondrial Cyt $b$ gene sequences $(1140 \mathrm{bp})$ were amplified by the forward primer CytBCapF (5'tggaatctaaccatgaccaatg-3') and reverse primer CytBCapR (5'-ggctattctccttttctggttt-3'). PCR amplification was conducted in $50 \mu \mathrm{L}$ volume, containing $25 \mu \mathrm{L}$ Kappa ready mix, $3 \mu \mathrm{L}$ DNA template, $1 \mu \mathrm{L}$ forward primer, $1 \mu \mathrm{L}$ reverse primer and $20 \mu \mathrm{L} \mathrm{ddH}_{2} \mathrm{O}$. The PCR amplification was conducted using a Infinigen Thermal Cycler based on program, initial denaturation at $94^{\circ} \mathrm{C}$ for $5 \mathrm{~min}$, followed by 35 cycles, each consisting of $30 \mathrm{sec}$ denaturation at $94^{\circ} \mathrm{C}, 45 \mathrm{sec}$ primers annealing at $49^{\circ} \mathrm{C}, 90 \mathrm{sec}$ elongation at $72^{\circ} \mathrm{C}$, then 5 min elongation at $72^{\circ} \mathrm{C}$ for the final stage and were stored at $4^{\circ} \mathrm{C}$. The PCR product was visualized using 1\% gel Agarose. Electrophoresis was run on $100 \mathrm{~V}$ condition for $20 \mathrm{~min}$ and the amplification result could be seen on UV light. The PCR products were sequenced by 1st BaseAsia, Malaysia. 


\section{Data Analysis}

Cyt $b$ gene sequence was analyzed using Clustal W (Thompson et al., 1994) in Molecular Evolutionary Genetics Analysis (MEGA6) program (Tamura et al., 2013). Alignment of Kacang and EG goat Cyt $b$ gene sequence was conducted through multiple alignment with $C$. hircus (D84201.1), C. aegagrus (AB004069.1) and C. falconeri (AB044309.1) as comparator which were obtained from GenBank. The nucleotide sequence then was translated in the form of amino acids by Mitochondrial Vertebrate genetic code.

\section{RESULTS AND DISCUSSION}

Cyt b gene amplification result was $1261 \mathrm{bp}$, so it can be obtained the complete Cyt $b$ gene sequences throughout $1140 \mathrm{bp}$ and 377 amino acids that begin with start codon (ATG) and end with stop codon (AGA). This is consistent with the results of some research that stated the length of Cyt b gene in goat was $1140 \mathrm{bp}$ and preceded with start codon that encoded by ATG and end with stop codon that encoded by AGA ( Pietro et al., 2003; Guang-Xin et al., 2015). Comparison result showed, there were differences in the characteristics of the amino acids.

The difference of the amino acid sequences within Kacang and EG goat (Table 1-4) showed one synonymous amino acids (codon site $164^{\text {th }}$ ), two non synonymous amino acids (site codon $16^{\text {th }}$ (KG4, EG6, EG11, EG12) and 231 $1^{\text {st }}$ (EG7), and 374 conserve amino acids. A comparison of the amino acid sequences of Kacang and EG goat with $C$. hircus (Table 1-2) showed 13 synonymous amino acids (codon site $16^{\text {th }}$ (except KG4, EG6, EG11, EG12), 102 $2^{\text {th }}, 131^{\text {st }}, 132^{\text {nd }}, 146^{\text {th }}, 148^{\text {th }}$, $149^{\text {th }}, 164^{\text {th }}, 197^{\text {th }}, 231^{\text {st }}$ (except forEG7), 240 $0^{\text {th }}$, $283^{\text {rd }}$ and $\left.355^{\text {th }}\right)$, four non synonymous amino acids (site codon 16th ${ }^{\text {th }}$ (only for KG4, EG6, EG11, EG12), 190 $\left.{ }^{\text {th }}, 214^{\text {th }}, 231^{\text {st }}\right)$ and 362 conserve amino acids. While comparison of Kacang and EG goats with C. aegagrus (Table 34) showed seven synonymous amino acids (codon site $16^{\text {th }}$ (only for KG4, EG6. EG11. EG12); 102 ${ }^{\text {nd }}$, $164^{\text {th }}, 197^{\text {th }}, 231^{\text {st }}$ (except for EG7); 283 ${ }^{\text {th }}$ and $355^{\text {th }}$ ) and three non synonymous amino acids (codon site $16^{\text {th }}$ (except for KG4, EG6, EG11, EG12), 214 $4^{\text {th }}$ and $231^{\text {st }}$ (only for EG7)) and 369 conserve amino acids. Major differences were between Kacang and EG goats with $C$. falconeri (data not presented) with as many as 43 synonymous amino acids (codon site $5^{\text {th }}, 20^{\text {th }}, 25^{\text {th }}$, $37^{\text {th }}, 41^{\text {st }}, 43^{\text {th }}, 62^{\text {nd }}, 72^{\text {nd }}, 77^{\text {th }}, 95^{\text {th }}, 102^{\text {nd }}, 105^{\text {th }}$, $121^{\text {st }}, 123^{\text {rd }}, 131^{\text {st }}, 139^{\text {th }}, 149^{\text {th }}, 164^{\text {th }}, 165^{\text {th }}, 170^{\text {th }}$, $173^{\text {rd }}, 188^{\text {th }}, 196^{\text {th }}, 197^{\text {th }}, 204^{\text {th }}, 206^{\text {th }}, 215^{\text {th }}, 229^{\text {th }}$, $232^{\text {nd }}, 246^{\text {th }}, 259^{\text {th }}, 266^{\text {th }}, 267^{\text {th }}, 272^{\text {nd }}, 283^{\text {th }}, 304^{\text {th }}$, $325^{\text {th }}, 334^{\text {th }}, 355^{\text {th }}, 357^{\text {th }}, 367^{\text {th }}, 373^{\text {rd }}$ and $\left.375^{\text {th }}\right)$ and six non synonymous amino acids (codon site $16^{\text {th }}$, $97^{\text {th }}, 189^{\text {th }}, 214^{\text {th }}, 231^{\text {st }}$ and $303^{\text {rd }}$ ) and 328 conserve amino acids. Conserve amino acid is the most found in this study. The present result is consistent with the character of Cyt b gene that it is converse, does not change much or does not experience mutation, thus it will be more sensitive to be used as genetic marker for identifying species purity, grouping based on breed and determining genetic relationship (Manceau et al., 1999; Widayanti et al., 2006).

Amino acid sequence of Kacang and EG goats had least difference with $C$. aegagrus (Table 5 and Table 6). There were three amino acids at codon site $17^{\text {th }}$ (except sample KG4, EG6, EG11 and EG12) and 215 ${ }^{\text {th }}$ (Threonine turned Alanine), and site codon 232 $2^{\text {nd }}$ (sample EG7) Alanine turned Valine. The differences of amino acid between Kacang, EG and C. hircus were four amino acids at codon site $17^{\text {th }}$ (sample KG4, EG6, EG11 and EG12) that Alanine turned Treonine, 191 ${ }^{\text {st }}$ (Glycine turned Alanine) and $215^{\text {th }}$ (Threonine turned Alanine), and site codon $232^{\text {nd }}$ (sample EG7) that Alanine turned Valine. When compared between Kacang and EG to C. falconeri, there were 5 differences of amino acid. It was at codon site $17^{\text {th }}$ (sample KG4, EG6, EG11 and EG12) that Alanine turned Threonine, $98^{\text {th }}$ (Valine turned Isoleucine), $190^{\text {th }}$ (Alanine turned Threonine), $215^{\text {th }}$ (Threonine turned Alanine), 232 $2^{\text {nd }}$ (only sample EG7) that Alanine turned Valine and $304^{\text {th }}$ (Methionine turned Valine). There were two specific amino acid differences in Kacang and EG goat on codon site $191^{\text {st }}$ and $215^{\text {th }}$ when compared to $C$. hircus, and on codon site $17^{\text {th }}$ and $215^{\text {th }}$ when compared with C. aegagrus. While there were four spesific amino acid differences in Kacang and EG goat when compared to $C$. falconeri, there were on codon site $98^{\text {th }}, 190^{\text {th }}, 215^{\text {th }}$ and $304^{\text {th }}$. It differences can be used as genetic markers to distinguish Kacang and EG goat with $C$. hircus, C. aegagrus and C. falconeri, but it cannot be used as a genetic marker for level intra-species to distinguish Kacang and EG goat. Based on the number of amino acid differences that can be presumed that Kacang and EG goat have a closer genetic relationship with $C$. aegagrus compared to $C$. hircus and $C$. falconeri. C. aegagrus (Bezoar) is a wild goat which is the ancestor of 
Table 1. Difference in Codon Site of Amino Acid among Kacang and EG Goats with C. hircus

\begin{tabular}{|c|c|c|c|c|c|c|c|c|c|c|c|c|c|c|c|}
\hline \multirow{2}{*}{ Sample } & \multicolumn{15}{|c|}{ Codon site on } \\
\hline & 16 & 102 & 131 & 132 & 146 & 148 & 149 & 164 & 190 & 197 & 214 & 231 & 240 & 283 & 355 \\
\hline C.hircus* & GCA & TAT & GTC & CTA & $\mathrm{ACC}$ & CTC & CTC & TGA & GGC & CTC & ACA & GCC & CTA & ATC & ATT \\
\hline KG1 & $\ldots$ &.. $\mathrm{C}$ &.. $\mathrm{T}$ & T.. &.. $\mathrm{T}$ &.. $\mathrm{T}$ &.. $\mathrm{T}$ & $\ldots$ & .C. &.. $\mathrm{T}$ & G.. & $\ldots$ & T.. &.. $\mathrm{T}$ &.. $\mathrm{C}$ \\
\hline KG2 & $\ldots$ &.. $\mathrm{C}$ &.. $\mathrm{T}$ & T.. &.. $\mathrm{T}$ &.. $\mathrm{T}$ &.. $\mathrm{T}$ & $\ldots$ & .C. &.. $\mathrm{T}$ & G.. & $\ldots$ & T.. &.. $\mathrm{T}$ &.. $\mathrm{C}$ \\
\hline KG3 & $\ldots$ &.. $\mathrm{C}$ &.. $\mathrm{T}$ & T.. &.. $\mathrm{T}$ &.. $\mathrm{T}$ &.. $\mathrm{T}$ & $\ldots$ & .C. &.. $\mathrm{T}$ & G.. & $\ldots$ & T.. &.. $\mathrm{T}$ &.. $\mathrm{C}$ \\
\hline KG4 & A.. &.. $\mathrm{C}$ &.. $\mathrm{T}$ & T.. &.. $\mathrm{T}$ &.. $\mathrm{T}$ &.. $\mathrm{T}$ & . & .C. &.. $\mathrm{T}$ & G.. & $\ldots$ & T.. &.. $\mathrm{T}$ & ..C \\
\hline KG5 & $\ldots$ &.. $\mathrm{C}$ &.. $\mathrm{T}$ & T.. &.. $\mathrm{T}$ &.. $\mathrm{T}$ &.. $\mathrm{T}$ & $\ldots$ & .C. &.. $\mathrm{T}$ & G.. & $\ldots$ & T.. &.. $\mathrm{T}$ &.. $\mathrm{C}$ \\
\hline KG6 & $\cdots$ &.. $\mathrm{C}$ &.. $\mathrm{T}$ & T.. &.. $\mathrm{T}$ &.. $\mathrm{T}$ &.. $\mathrm{T}$ & $\ldots$ & .C. &.. $\mathrm{T}$ & G.. & $\ldots$ & T.. &.. $\mathrm{T}$ &..$C$ \\
\hline KG7 & $\ldots$ &.. $\mathrm{C}$ &.. $\mathrm{T}$ & $\mathrm{T} .$. &.. $\mathrm{T}$ &.. $\mathrm{T}$ &.. $\mathrm{T}$ &.. $\mathrm{G}$ & .C. &.. $\mathrm{T}$ & G.. & $\ldots$ & T.. &.. $\mathrm{T}$ & ..C \\
\hline KG8 & $\ldots$ &..$C$ &.. $\mathrm{~T}$ & T.. &.. $\mathrm{T}$ &.. $\mathrm{T}$ &.. $\mathrm{T}$ & $\ldots$ & .C. &.. $\mathrm{T}$ & G.. & $\ldots$ & T.. &.. $\mathrm{T}$ &..$C$ \\
\hline KG9 & $\ldots$ &..$C$ &.. $\mathrm{~T}$ & T.. &.. $\mathrm{T}$ &.. $\mathrm{T}$ &.. $\mathrm{T}$ & $\ldots$ & .C. &.. $\mathrm{T}$ & G.. & $\ldots$ & T.. &.. $\mathrm{T}$ &.. $\mathrm{C}$ \\
\hline KG10 & $\ldots$ &.. $\mathrm{C}$ &.. $\mathrm{T}$ & T.. &.. $\mathrm{T}$ &.. $\mathrm{T}$ &.. $\mathrm{T}$ & $\ldots$ & .C. &.. $\mathrm{T}$ & G.. & $\ldots$ & T.. &.. $\mathrm{T}$ &.. $\mathrm{C}$ \\
\hline KG11 & $\ldots$ &.. $\mathrm{C}$ &.. $\mathrm{T}$ & T.. &.. $\mathrm{T}$ &.. $\mathrm{T}$ &.. $\mathrm{T}$ & $\ldots$ & .C. &.. $\mathrm{T}$ & G.. & $\ldots$ & T.. &.. $\mathrm{T}$ & ..C \\
\hline KG12 & $\ldots$ &.. $\mathrm{C}$ &.. $\mathrm{T}$ & T.. &.. $\mathrm{T}$ &.. $\mathrm{T}$ &.. $\mathrm{T}$ & $\ldots$ & .C. &.. $\mathrm{T}$ & G.. & $\ldots$ & T.. &.. $\mathrm{T}$ &..$C$ \\
\hline KG13 & $\ldots$ &..$C$ &.. $\mathrm{~T}$ & T.. &.. $\mathrm{T}$ &.. $\mathrm{T}$ &..$T$ & $\ldots$ & .C. &.. $\mathrm{T}$ & G.. & $\ldots$ & T.. &.. $\mathrm{T}$ &..$C$ \\
\hline KG14 & $\ldots$ &.. $\mathrm{C}$ &.. $\mathrm{T}$ & T.. &.. $\mathrm{T}$ &.. $\mathrm{T}$ &.. $\mathrm{T}$ &.. $\mathrm{G}$ & .C. &.. $\mathrm{T}$ & G.. & $\ldots$ & T.. &.. $\mathrm{T}$ & ..C \\
\hline KG15 & $\ldots$ &.. $\mathrm{C}$ &.. $\mathrm{T}$ & T.. &.. $\mathrm{T}$ &.. $\mathrm{T}$ &.. $\mathrm{T}$ & $\ldots$ & .C. &.. $\mathrm{T}$ & G.. & $\ldots$ & T.. &.. $\mathrm{T}$ &.. $\mathrm{C}$ \\
\hline KG16 & $\ldots$ &..$C$ &.. $\mathrm{~T}$ & T.. &.. $\mathrm{T}$ &.. $\mathrm{T}$ &.. $\mathrm{T}$ &.. $\mathrm{G}$ & .C. &.. $\mathrm{T}$ & G.. & $\ldots$ & T.. &.. $\mathrm{T}$ & ..C \\
\hline KG17 & $\ldots$ &.. $\mathrm{C}$ &.. $\mathrm{T}$ & T.. &.. $\mathrm{T}$ &.. $\mathrm{T}$ &.. $\mathrm{T}$ & $\ldots$ & .C. &..$T$ & G.. & $\ldots$ & T.. &.. $\mathrm{T}$ &.. $\mathrm{C}$ \\
\hline KG18 & $\ldots$ &.. $\mathrm{C}$ &.. $\mathrm{T}$ & T.. &.. $\mathrm{T}$ &.. $\mathrm{T}$ &.. $\mathrm{T}$ & $\ldots$ & .C. &.. $\mathrm{T}$ & G.. & $\ldots$ & T.. &.. $\mathrm{T}$ & ..C \\
\hline KG19 & $\ldots$ &.. $\mathrm{C}$ &.. $\mathrm{T}$ & T.. &.. $\mathrm{T}$ &.. $\mathrm{T}$ &.. $\mathrm{T}$ & $\ldots$ & .C. &.. $\mathrm{T}$ & G.. & $\ldots$ & T.. &.. $\mathrm{T}$ &.. $\mathrm{C}$ \\
\hline EG1 & $\ldots$ &..$C$ &.. $\mathrm{~T}$ & T.. &.. $\mathrm{T}$ &.. $\mathrm{T}$ &.. $\mathrm{T}$ & $\ldots$ & .C. &.. $\mathrm{T}$ & G.. & $\ldots$ & T.. &.. $\mathrm{T}$ &..$C$ \\
\hline EG2 & $\ldots$ &.. $\mathrm{C}$ &.. $\mathrm{T}$ & T.. &.. $\mathrm{T}$ &.. $\mathrm{T}$ &.. $\mathrm{T}$ & $\ldots$ & .C. &.. $\mathrm{T}$ & G.. & $\ldots$ & T.. &.. $\mathrm{T}$ &.. $\mathrm{C}$ \\
\hline EG3 & $\ldots$ &.. $\mathrm{C}$ &.. $\mathrm{T}$ & T.. &.. $\mathrm{T}$ &.. $\mathrm{T}$ &.. $\mathrm{T}$ & $\ldots$ & .C. &.. $\mathrm{T}$ & G.. & $\ldots$ & T. &.. $\mathrm{T}$ & ..C \\
\hline EG4 & $\ldots$ &..$C$ &.. $\mathrm{~T}$ & T.. &.. $\mathrm{T}$ &.. $\mathrm{T}$ &.. $\mathrm{T}$ & $\ldots$ & .C. &..$T$ & G.. & $\ldots$ & T.. &.. $\mathrm{T}$ &..$C$ \\
\hline EG5 & $\ldots$ &.. $\mathrm{C}$ &.. $\mathrm{T}$ & T.. &.. $\mathrm{T}$ &.. $\mathrm{T}$ &.. $\mathrm{T}$ & $\ldots$ & .C. &..$T$ & G.. & $\ldots$ & T.. &.. $\mathrm{T}$ &.. $\mathrm{C}$ \\
\hline EG6 & A.. &.. $\mathrm{C}$ &.. $\mathrm{T}$ & T.. &.. $\mathrm{T}$ &.. $\mathrm{T}$ &.. $\mathrm{T}$ & $\ldots$ & .C. &.. $\mathrm{T}$ & G.. & $\ldots$ & T.. &.. $\mathrm{T}$ & ..C \\
\hline EG7 & $\ldots$ &.. $\mathrm{C}$ &.. $\mathrm{T}$ & T.. &.. $\mathrm{T}$ &.. $\mathrm{T}$ &.. $\mathrm{T}$ & $\cdots$ & .C. &.. $\mathrm{T}$ & G.. & .T. & T.. &.. $\mathrm{T}$ &.. $\mathrm{C}$ \\
\hline EG8 & $\ldots$ &..$C$ &.. $\mathrm{~T}$ & T.. &.. $\mathrm{T}$ &.. $\mathrm{T}$ &..$T$ & $\ldots$ & .C. &.. $\mathrm{T}$ & G.. & $\ldots$ & T.. &.. $\mathrm{T}$ &.. $\mathrm{C}$ \\
\hline EG9 & $\ldots$ &.. $\mathrm{C}$ &.. $\mathrm{T}$ & T.. &.. $\mathrm{T}$ &.. $\mathrm{T}$ &.. $\mathrm{T}$ &.. $\mathrm{G}$ & .C. &.. $\mathrm{T}$ & G.. & $\ldots$ & T.. &.. $\mathrm{T}$ &.. $\mathrm{C}$ \\
\hline EG10 & $\ldots$ &.. $\mathrm{C}$ &.. $\mathrm{T}$ & T.. &.. $\mathrm{T}$ &.. $\mathrm{T}$ &.. $\mathrm{T}$ &.. $\mathrm{G}$ & .C. &.. $\mathrm{T}$ & G.. & $\ldots$ & T.. &.. $\mathrm{T}$ & ..C \\
\hline EG11 & A.. &..$C$ &.. $\mathrm{~T}$ & T.. &.. $\mathrm{T}$ &.. $\mathrm{T}$ &.. $\mathrm{T}$ & $\ldots$ & .C. &.. $\mathrm{T}$ & G.. & $\ldots$ & T.. &.. $\mathrm{T}$ &..$C$ \\
\hline EG12 & A.. &..$C$ &.. $\mathrm{~T}$ & T.. &.. $\mathrm{T}$ &.. $\mathrm{T}$ &.. $\mathrm{T}$ & $\ldots$ & .C. &.. $\mathrm{T}$ & G.. & $\ldots$ & T.. &.. $\mathrm{T}$ &..$C$ \\
\hline
\end{tabular}

* from GenBank; KG: Kacang goat; EG: Ettawa Grade goat

(.) same with $C$. hircus

Codon (Nucleotide base) :A (Adenine), C (Citosine), G (Guanine), T (Timine) 
Table 2. Differences in Amino Acid Site among Kacang and EG Goats with C. hircus

\begin{tabular}{|c|c|c|c|c|c|c|c|c|c|c|c|c|c|c|c|}
\hline \multirow{2}{*}{ Sample } & \multicolumn{15}{|c|}{ Amino Acid Site on } \\
\hline & 16 & 102 & 131 & 132 & 146 & 148 & 149 & 164 & 190 & 197 & 214 & 231 & 240 & 283 & 355 \\
\hline C. hircus* & $\mathrm{A}$ & $\mathrm{Y}$ & $\mathrm{V}$ & $\mathrm{L}$ & $\mathrm{T}$ & $\mathrm{L}$ & $\mathrm{L}$ & $\mathrm{W}$ & G & $\mathrm{L}$ & $\mathrm{T}$ & $\mathrm{A}$ & $\mathrm{L}$ & I & $\mathrm{I}$ \\
\hline KG1 & . & . & . & . & . & . & . & . & A & . & $\mathrm{A}$ & . & . & . & . \\
\hline KG2 & . & . & . & . & . & . & . & . & A & . & A & . & . & . & . \\
\hline KG3 & . & . & . & . & . & . & . & . & A & . & A & . & . & . & . \\
\hline KG4 & $\mathrm{T}$ & . & . & . & . & . & . & . & A & . & $\mathrm{A}$ & . & . & . & . \\
\hline KG5 & . & . & . & . & . & . & . & . & A & . & A & . & . & . & . \\
\hline KG6 & . & . & . & . & . & . & . & . & A & . & A & . & . & . & . \\
\hline KG7 & . & . & . & . & . & . & . & . & A & . & A & . & . & . & . \\
\hline KG8 & . & . & . & . & . & . & . & . & A & . & A & . & . & . & . \\
\hline KG9 & . & . & . & . & . & . & . & . & A & . & A & . & . & . & . \\
\hline KG10 & . & . & . & . & . & . & . & . & A & . & A & . & . & . & . \\
\hline KG11 & . & . & . & . & . & . & . & . & A & . & A & . & . & . & . \\
\hline KG12 & . & . & . & . & . & . & . & . & A & . & A & . & . & . & . \\
\hline KG13 & . & . & . & . & . & . & . & . & A & . & A & . & . & . & . \\
\hline KG14 & . & . & . & . & . & . & . & . & A & . & A & . & . & . & . \\
\hline KG15 & . & . &. & . & . & . & . & . & A & . & A & . & . & . & . \\
\hline KG16 & . & . & . & . & . & . & . & . & A & . & A & . & . & . & . \\
\hline KG17 & . & . & . & . & . & . & . & . & A & . & A & . & . & . & . \\
\hline KG18 & . & . & . & . & . & . & . & . & A & . & A & . & . & . & . \\
\hline KG19 & . & . & . & . & . & . & . & . & A & . & A & . & . & . & . \\
\hline EG1 & . & . & . & . & . & . & . & . & A & . & A & . & . & . & . \\
\hline EG2 & . & . &. & . & . & . & . & . & A & . & A & . & . & . & . \\
\hline EG3 & . & . &. & . & . & . & . & . & A & . & A & . & . & . & . \\
\hline EG4 & . & . & . & . & . & . & . & . & A & . & A & . & . & . & . \\
\hline EG5 & . & . & . & . & . & . & . & . & A & . & A & . & . & . & . \\
\hline EG6 & $\mathrm{T}$ & . &. & . & . & . & . & . & A & . & A & . & . & . & . \\
\hline EG7 & . & . & . & . & . & . & . & . & A & . & A & V & . & . & . \\
\hline EG8 & . & . & . & . & . & . & . & . & A & . & A & . & . & . & . \\
\hline EG9 & . & . & . & . & . & . & . & . & A & . & A & . & . & . & . \\
\hline EG10 & . & . &. & . & . & . & . & . & A & . & A & . & . & . & . \\
\hline EG11 & $\mathrm{T}$ & . & . & . & . & . & . & . & A & . & A & . & . & . & . \\
\hline EG12 & $\mathrm{T}$ & . & . & . & . & . & . & . & A & . & A & . & . & . & . \\
\hline
\end{tabular}

* goat breed was taken from GenBank; KG: Kacang goat; EG: Ettawa Grade goat (.) same with $C$. hircus

Amino acid :A (Alanine), G (Glycine), I (Isoleucine), L (Leucine), T (Threonine), V (Valine), W (Tryptophan), Y (Tyrosine) 
Table 3. Differences Codon Site of Amino Acid among Kacang and EG Goats with C. aegagrus

\begin{tabular}{|c|c|c|c|c|c|c|c|c|}
\hline \multirow{2}{*}{ Sample } & \multicolumn{8}{|c|}{ Codon site on } \\
\hline & 16 & 102 & 164 & 197 & 214 & 231 & 283 & 355 \\
\hline C. aegagrus* & $\mathrm{ACA}$ & TAT & TGA & CTC & ACA & GCC & ATC & ATT \\
\hline KG1 & G.. &.. $\mathrm{C}$ & $\ldots$ &.. $\mathrm{T}$ & G.. & $\ldots$ &.. $\mathrm{T}$ &.. $\mathrm{C}$ \\
\hline KG2 & G.. &.. $\mathrm{C}$ & $\ldots$ &.. $\mathrm{T}$ & G.. & $\ldots$ &.. $\mathrm{T}$ &.. $\mathrm{C}$ \\
\hline KG3 & G.. &.. $\mathrm{C}$ & $\ldots$ &.. $\mathrm{T}$ & G.. & $\ldots$ &.. $\mathrm{T}$ &.. $\mathrm{C}$ \\
\hline KG4 & $\ldots$ &.. $\mathrm{C}$ & $\ldots$ &.. $\mathrm{T}$ & G.. & $\ldots$ &.. $\mathrm{T}$ &.. $\mathrm{C}$ \\
\hline KG5 & G.. &.. $\mathrm{C}$ & $\ldots$ &.. $\mathrm{T}$ & G.. & $\ldots$ &.. $\mathrm{T}$ &.. $\mathrm{C}$ \\
\hline KG6 & G.. &.. $\mathrm{C}$ & $\ldots$ &.. $\mathrm{T}$ & G.. & $\ldots$ &.. $\mathrm{T}$ &.. $\mathrm{C}$ \\
\hline KG7 & G.. &.. $\mathrm{C}$ &.. $\mathrm{G}$ &.. $\mathrm{T}$ & G.. & $\ldots$ &.. $\mathrm{T}$ &.. $\mathrm{C}$ \\
\hline KG8 & G.. &.. $\mathrm{C}$ & $\ldots$ &.. $\mathrm{T}$ & G.. & $\ldots$ &.. $\mathrm{T}$ &.. $\mathrm{C}$ \\
\hline KG9 & G.. &.. $\mathrm{C}$ & $\ldots$ &.. $\mathrm{T}$ & G.. & $\ldots$ &.. $\mathrm{T}$ &.. $\mathrm{C}$ \\
\hline KG10 & G.. &.. $\mathrm{C}$ & $\ldots$ &.. $\mathrm{T}$ & G.. & $\ldots$ &.. $\mathrm{T}$ &.. $\mathrm{C}$ \\
\hline KG11 & G.. &.. $\mathrm{C}$ & $\ldots$ &.. $\mathrm{T}$ & G.. & $\ldots$ &.. $\mathrm{T}$ &.. $\mathrm{C}$ \\
\hline KG12 & G.. &.. $\mathrm{C}$ & $\ldots$ &.. $\mathrm{T}$ & G.. & $\ldots$ &.. $\mathrm{T}$ &.. $\mathrm{C}$ \\
\hline KG13 & G.. &..$C$ & $\ldots$ &.. $\mathrm{T}$ & G.. & $\ldots$ &.. $\mathrm{T}$ &..$C$ \\
\hline KG14 & G.. &.. $\mathrm{C}$ &.. $\mathrm{G}$ &.. $\mathrm{T}$ & G.. & $\ldots$ &.. $\mathrm{T}$ &.. $\mathrm{C}$ \\
\hline KG15 & G.. &..$C$ & $\ldots$ &.. $\mathrm{T}$ & G.. & $\ldots$ &.. $\mathrm{T}$ &..$C$ \\
\hline KG16 & G.. &.. $\mathrm{C}$ &.. $\mathrm{G}$ &.. $\mathrm{T}$ & G.. & $\ldots$ &.. $\mathrm{T}$ &.. $\mathrm{C}$ \\
\hline KG17 & G.. &..$C$ & $\ldots$ &.. $\mathrm{T}$ & G.. & $\ldots$ &.. $\mathrm{T}$ &.. $\mathrm{C}$ \\
\hline KG18 & G.. &.. $\mathrm{C}$ & $\ldots$ &.. $\mathrm{T}$ & G.. & $\ldots$ &.. $\mathrm{T}$ &.. $\mathrm{C}$ \\
\hline KG19 & G.. &.. $\mathrm{C}$ & $\ldots$ &.. $\mathrm{T}$ & G.. & $\ldots$ &.. $\mathrm{T}$ &.. $\mathrm{C}$ \\
\hline EG1 & G.. &..$C$ & $\ldots$ &.. $\mathrm{T}$ & G.. & $\ldots$ &.. $\mathrm{T}$ &..$C$ \\
\hline EG2 & G.. &.. $\mathrm{C}$ & $\ldots$ &.. $\mathrm{T}$ & G.. & $\ldots$ &.. $\mathrm{T}$ &.. $\mathrm{C}$ \\
\hline EG3 & G.. &..$C$ & $\ldots$ &.. $\mathrm{T}$ & G.. & $\ldots$ &.. $\mathrm{T}$ &.. $\mathrm{C}$ \\
\hline EG4 & G.. &.. $\mathrm{C}$ & $\ldots$ &.. $\mathrm{T}$ & G.. & $\ldots$ &.. $\mathrm{T}$ &.. $\mathrm{C}$ \\
\hline EG5 & G.. &.. $\mathrm{C}$ & $\ldots$ &.. $\mathrm{T}$ & G.. & $\ldots$ &.. $\mathrm{T}$ &.. $\mathrm{C}$ \\
\hline EG6 & $\ldots$ &..$C$ & $\ldots$ &.. $\mathrm{T}$ & G.. & $\ldots$ &.. $\mathrm{T}$ &.. $\mathrm{C}$ \\
\hline EG7 & G.. &.. $\mathrm{C}$ & $\ldots$ &.. $\mathrm{T}$ & G.. & .T. &.. $\mathrm{T}$ & ..C \\
\hline EG8 & G.. &.. $\mathrm{C}$ & $\ldots$ &.. $\mathrm{T}$ & G.. & $\ldots$ &.. $\mathrm{T}$ &.. $\mathrm{C}$ \\
\hline EG9 & G.. &.. $\mathrm{C}$ &.. $\mathrm{G}$ &.. $\mathrm{T}$ & G.. & $\ldots$ &.. $\mathrm{T}$ &.. $\mathrm{C}$ \\
\hline EG10 & G.. &..$C$ &.. $\mathrm{G}$ &.. $\mathrm{T}$ & G.. & $\ldots$ &.. $\mathrm{T}$ &..$C$ \\
\hline EG11 & $\ldots$ &.. $\mathrm{C}$ & $\ldots$ &.. $\mathrm{T}$ & G.. & $\ldots$ &.. $\mathrm{T}$ &.. $\mathrm{C}$ \\
\hline EG12 & $\ldots$ &..$C$ & $\ldots$ &.. $\mathrm{T}$ & G.. & $\ldots$ &.. $\mathrm{T}$ &..$C$ \\
\hline
\end{tabular}

* goat breed was taken from GenBank; KG: Kacang goat; EG: Ettawa Grade goat

(.) same with C. aegagrus

Codon (Nucleotide base) :A (Adenine), C (Citosine), G (Guanine), T (Timine) 
Table 4. Differences Amino Acid Site among Kacang and EG goats with C. aegagrus

\begin{tabular}{|c|c|c|c|c|c|c|c|c|}
\hline \multirow{2}{*}{ Sample } & \multicolumn{8}{|c|}{ Amino Acid Site on } \\
\hline & 16 & 102 & 164 & 197 & 214 & 231 & 283 & 355 \\
\hline C. aegagrus* & $\mathrm{T}$ & $\mathrm{Y}$ & $\mathrm{W}$ & $\mathrm{L}$ & $\mathrm{T}$ & $\mathrm{A}$ & I & $\mathrm{I}$ \\
\hline KG1 & $\mathrm{A}$ & . & . & . & $\mathrm{A}$ & . & . & . \\
\hline KG2 & $\mathrm{A}$ & . & . & . & $\mathrm{A}$ & . & . & . \\
\hline KG3 & $\mathrm{A}$ & . & . & . & $\mathrm{A}$ & . & . & . \\
\hline KG4 & . & . & . & . & $\mathrm{A}$ & . & . & . \\
\hline KG5 & $\mathrm{A}$ & . & . & . & $\mathrm{A}$ & . & . & . \\
\hline KG6 & A & . & . & . & $\mathrm{A}$ & . & . & . \\
\hline KG7 & A & . & . & . & A & . & . & . \\
\hline KG9 & A & . & . & . & $\mathrm{A}$ & . & . & . \\
\hline KG9 & $\mathrm{A}$ & . & . & . & $\mathrm{A}$ & . & . & . \\
\hline KG10 & A & . & . & . & A & . & . & . \\
\hline KG11 & A & . & . & . & A & . & . & . \\
\hline KG12 & $\mathrm{A}$ & . & . & . & A & . & . & . \\
\hline KG13 & A & . & . & . & A & . & . & . \\
\hline KG14 & A & . & . & . & A & . & . & . \\
\hline KG15 & A & . & . & . & A & . & . & . \\
\hline KG16 & $\mathrm{A}$ & . & . & . & A & . & . & . \\
\hline KG17 & A & . & . & . & A & . & . & . \\
\hline KG18 & A & . & . & . & A & . & . & . \\
\hline KG19 & $\mathrm{A}$ & . & . & . & A & . & . & . \\
\hline EG1 & A & . & . & . & $\mathrm{A}$ & . & . & . \\
\hline EG2 & A & . & . & . & A & . & . & . \\
\hline EG3 & A & . & . & . & A & . & . & . \\
\hline EG4 & $\mathrm{A}$ & . & . & . & A & . & . & . \\
\hline EG5 & A & . & . & . & A & . & . & . \\
\hline EG6 & . & . & . & . & A & . & . & . \\
\hline EG7 & $\mathrm{A}$ & . & . & . & A & V & . & . \\
\hline EG8 & A & . & . & . & $\mathrm{A}$ & . & . & . \\
\hline EG9 & A & . & . & . & A & . & . & . \\
\hline EG10 & A & . & . & . & A & . & . & . \\
\hline EG11 & . & . & . & . & A & . & . & . \\
\hline EG12 & . & . & . & . & A & . & . & . \\
\hline
\end{tabular}

* goat breed was taken from GenBank; KG: Kacang goat; EG: Ettawa Grade goat (.) same with C. aegagrus

Amino acid :A (Alanine), I (Isoleucine), L (Leucine), T (Threonine), V (Valine), W (Tryptophan), Y (Tyrosine) 
Table 5. Differences of Codon Site on Cytochrome b among Kacang and EG goat with Three Comparator Goats

\begin{tabular}{|c|c|c|c|c|c|c|c|}
\hline \multirow{2}{*}{ Sample } & \multicolumn{7}{|c|}{ Codon site on } \\
\hline & 16 & 97 & 189 & 190 & 214 & 231 & 303 \\
\hline C. hircus* & GCA & ATC & ACA & GGC & ACA & GCC & GTA \\
\hline C. aegagrus* & A.. & $\ldots$ & $\ldots$ & .C. & $\ldots$ & $\ldots$ & $\ldots$ \\
\hline C. falconeri* & $\ldots$ & G.. & G.. & .C. & $\ldots$ & $\ldots$ & A.. \\
\hline KG1 & $\ldots$ & $\ldots$ & $\ldots$ & .C. & G.. & $\ldots$ & $\ldots$ \\
\hline KG2 & $\ldots$ & $\ldots$ & $\ldots$ & .C. & G.. & $\ldots$ & $\ldots$ \\
\hline KG3 & $\ldots$ & $\ldots$ & $\ldots$ & .C. & G.. & $\ldots$ & $\ldots$ \\
\hline KG4 & A.. & $\ldots$ & $\ldots$ & .C. & G.. & $\ldots$ & $\ldots$ \\
\hline KG5 & $\ldots$ & $\ldots$ & $\ldots$ & .C. & G.. & $\ldots$ & $\ldots$ \\
\hline KG6 & $\ldots$ & $\ldots$ & $\ldots$ & .C. & G.. & $\ldots$ & $\ldots$ \\
\hline KG7 & $\ldots$ & $\ldots$ & $\ldots$ & .C. & G.. & $\ldots$ & $\ldots$ \\
\hline KG8 & $\ldots$ & $\ldots$ & $\ldots$ & .C. & G.. & $\ldots$ & $\ldots$ \\
\hline KG9 & $\ldots$ & $\ldots$ & $\ldots$ & .C. & G.. & $\ldots$ & $\ldots$ \\
\hline KG10 & $\ldots$ & $\ldots$ & $\ldots$ & .C. & G.. & $\ldots$ & $\ldots$ \\
\hline KG11 & $\ldots$ & $\ldots$ & $\ldots$ & .C. & G.. & $\ldots$ & $\ldots$ \\
\hline KG12 & $\ldots$ & $\ldots$ & $\ldots$ & .C. & G.. & $\ldots$ & $\ldots$ \\
\hline KG13 & $\ldots$ & $\ldots$ & $\ldots$ & .C. & G.. & $\ldots$ & $\ldots$ \\
\hline KG14 & $\ldots$ & $\ldots$ & $\ldots$ & .C. & G.. & $\ldots$ & $\ldots$ \\
\hline KG15 & $\ldots$ & $\ldots$ & $\ldots$ & .C. & G.. & $\ldots$ & $\ldots$ \\
\hline KG16 & $\ldots$ & $\ldots$ & $\ldots$ & .C. & G.. & $\ldots$ & $\ldots$ \\
\hline KG17 & $\ldots$ & $\ldots$ & $\ldots$ & .C. & G.. & $\ldots$ & $\ldots$ \\
\hline KG18 & $\ldots$ & $\ldots$ & $\ldots$ & .C. & G.. & $\ldots$ & $\ldots$ \\
\hline KG19 & $\ldots$ & $\ldots$ & $\ldots$ & .C. & G.. & $\ldots$ & $\ldots$ \\
\hline EG1 & $\ldots$ & $\ldots$ & $\ldots$ & .C. & G.. & $\ldots$ & $\ldots$ \\
\hline EG2 & $\ldots$ & $\ldots$ & $\ldots$ & .C. & G.. & $\ldots$ & $\ldots$ \\
\hline EG3 & $\ldots$ & $\ldots$ & $\ldots$ & .C. & G.. & $\ldots$ & $\ldots$ \\
\hline EG4 & $\ldots$ & $\ldots$ & $\ldots$ & .C. & G.. & $\ldots$ & $\ldots$ \\
\hline EG5 & $\ldots$ & $\ldots$ & $\ldots$ & .C. & G.. & $\ldots$ & $\ldots$ \\
\hline EG6 & A.. & $\ldots$ & $\ldots$ & .C. & G.. & $\ldots$ & $\ldots$ \\
\hline EG7 & $\ldots$ & $\ldots$ & $\ldots$ & .C. & G.. & .T. & $\ldots$ \\
\hline EG8 & $\ldots$ & $\ldots$ & $\ldots$ & .C. & G.. & $\ldots$ & $\ldots$ \\
\hline EG9 & $\ldots$ & $\ldots$ & $\ldots$ & .C. & G.. & $\ldots$ & $\ldots$ \\
\hline EG10 & $\ldots$ & $\ldots$ & $\ldots$ & .C. & G.. & $\ldots$ & $\ldots$ \\
\hline EG11 & A.. & $\ldots$ & $\ldots$ & .C. & G.. & $\ldots$ & $\ldots$ \\
\hline EG12 & A.. & $\ldots$ & $\ldots$ & .C. & G.. & $\ldots$ & $\ldots$ \\
\hline
\end{tabular}

* goat breeds were taken from GenBank; KG: Kacang goat; EG: Ettawa Grade goat (.) same with C. hircus

Codon (Nucleotide base) :A (Adenine), C (Citosine), G (Guanine), T (Timine) 
Table 6. Differences of Amino Acid Site on Cytochrome b among Kacang and EG goats with Three Comparator Goats

\begin{tabular}{|c|c|c|c|c|c|c|c|}
\hline \multirow{2}{*}{ Sample } & \multicolumn{7}{|c|}{ Amino Acid Site on } \\
\hline & 16 & 97 & 189 & 190 & 214 & 231 & 303 \\
\hline C. hircus* & $\mathrm{A}$ & $\mathrm{I}$ & $\mathrm{T}$ & $\mathrm{G}$ & $\mathrm{T}$ & & $\mathrm{V}$ \\
\hline C. aegagrus* & $\mathrm{T}$ & . & . & A & . & & . \\
\hline C. falconeri* & . & $\mathrm{V}$ & A & A & . & & M \\
\hline KG1 & . & . & . & A & A & & . \\
\hline KG2 & . & . & . & A & A & & . \\
\hline KG3 & . & . & . & $\mathrm{A}$ & A & & . \\
\hline KG4 & $\mathrm{T}$ & . & . & A & A & & . \\
\hline KG5 & . & . & . & $\mathrm{A}$ & $\mathrm{A}$ & & . \\
\hline KG6 & . & . & . & A & A & & . \\
\hline KG7 & . & . & . & $\mathrm{A}$ & A & & . \\
\hline KG8 & . & . & . & A & A & & . \\
\hline KG9 & . & . & . & A & A & & . \\
\hline KG10 & . & . & . & A & A & & . \\
\hline KG11 & . & . & . & A & A & & . \\
\hline $\mathrm{KG} 12$ & . & . & . & A & A & & . \\
\hline KG13 & . & . & . & A & A & & . \\
\hline KG14 & . & . & . & A & A & & . \\
\hline KG15 & . & . & . & A & A & & . \\
\hline KG16 & . & . & . & A & A & & . \\
\hline KG17 & . & . & . & A & A & & . \\
\hline KG18 & . & . & . & A & A & & . \\
\hline KG19 & . & . & . & A & A & & . \\
\hline EG1 & . & . & . & A & A & . & . \\
\hline EG2 & . & . & . & A & A & . & . \\
\hline EG3 & . & . & . & A & A & . & . \\
\hline EG4 & . & . & . & A & A & . & . \\
\hline EG5 & . & . & . & A & A & . & . \\
\hline EG6 & $\mathrm{T}$ & . & . & A & A & . & . \\
\hline EG7 & . & . & . & A & A & V & . \\
\hline EG8 & . & . & . & A & A & . & . \\
\hline EG9 & . & . & . & A & A & . & . \\
\hline EG10 & . & . & . & A & A & . & . \\
\hline EG11 & $\mathrm{T}$ & . & . & A & A & . & . \\
\hline EG12 & $\mathrm{T}$ & . & . & A & A & . & . \\
\hline
\end{tabular}

* goat breed was taken from GenBank; KG: Kacang goat; EG: Ettawa Grade goat (.) same withC. hircus

Amino acid :A (Alanine), G (Glycine), I (Isoleucine), M (Methionine), T (Threonine), V (Valine) 
Table 7. Amino Acid Characteristic on Cytochrome b Gene of Kacang and Ettawa Grade (EG) Goats Compared to 3 Comparator Goats

\begin{tabular}{lcccc}
\hline \multirow{2}{*}{ Characteristic } & Within Kacang and & \multicolumn{2}{c}{ Kacang and EG Goats Compared to } \\
\cline { 3 - 5 } & EG Goats & C. hircus & C. aegagrus & C. falconeri \\
\hline Synonymous amino acid & 1 & 11 & 5 & 43 \\
Non synonymous amino acid & 2 & 4 & 3 & 6 \\
Converse amino acid & 374 & 362 & 369 & 328 \\
Codon mutation & 1 & & 2 & 6 \\
$-1^{\text {st }}$ codon & 1 & 4 & 1 & 1 \\
$-2^{\text {nd }}$ codon & 1 & 2 & 5 & 42 \\
$-3^{\text {rd }}$ codon & & 9 & & \\
\hline
\end{tabular}

the domestic goat and C. falconeri (Markhor), and it has contributes to several species of goats in Asia (Mason, 1984; Takada et al., 1997; Pidancier et al., 2006). Naderi et al. (2008) stated that an early domestication center of C. aegagrus was in the Central Iranian Plateau (Yazd and Kerman Provinces) and in the Southern Zagros (Fars Province). While $C$. hircus (domestic goat) is a domestication goat of wild goat (C. aegagrus), which distribute to Africa (South Africa), Slovenia, Europe (Switzerland), Asia (Malaysia). According to Shackleton (1997), the distribution of $C$. aegagrus were include Afghanistan, Armenia, Azerbaijan (Nakhichevan), Lebanon (extinct), Russia (East Caucasus), Turkey, Georgia and Iran while distribution of C. falconeri were include India and Pakistan.

The most codon mutations occurred in third, second and first codon (Table 7). This is because the first and second codon in Cyt b gene b had a low gamma value, so the possibility of substitution mutation was very low. Conversely, the third codon had a high gamma value with the result that the possibility of substitution mutation is high (Farias et al., 2001). However, despite the third codon have a high substitution value, mostly substitution is saturation effect. Saturation effect is a change of nucleotide bases that are not followed by amino acid changes, which itis called synonymous amino acids (Kocher et al., 1989). Slechtova et al. (2006) and Doadrio and Perdices (2006) stated that the most varied nucleotide was at third codon, whereas most conserve nucleotides were at second codon.

\section{CONCLUSION}

Specific amino acids in Cyt $\mathrm{b}$ gene can be used as a genetic marker among KG and EG goat with 3 goat comparator. There were several specific amino acid that distinguish Kacang and EG goat with $C$. hircus, C. aegagrus and $C$. falconeri. But there were not amino acids can be used as a genetic marker to distinguish between Kacang and EG goat. Mutations in Cyt $b$ gene mostly were in third codon and the most differences were found between kacang and $E G$ goat with $C$. falconeri.

\section{ACKNOWLEGMENTS}

This study was funded by Pendidikan Magister Menuju Doktor untuk Sarjana Unggul (PMDSU) Scholarship of Bath II 2015, Ministry of Research, Technology and Higher Education Republic of Indonesia.

\section{REFERENCES}

Anderson, S., A. T. Bankier, B. G. Barell, M. H. L. de Bruijn, A. R. Coulson, J. Drouin, I. C. Eperon, D. P. Nierlich, B. A. Roe, F. Sanger, P. H. Schreier, R. Staden and I. G. Young. 1981. Sequence and the organization of the human mitochondrial genome. Nature 290:457-465. 
Batubara, A., M. Doloksaribu and B. Tiesnamurti. 2009. Potensi keragaman sumberdaya genetik kambing lokal Indonesia. Proceedings, Lokakarya Nasional Pengelolaan dan Perlindungan Sumber Daya Genetik di Indonesia: Manfaat Ekonomi untuk Mewujudkan Ketahanan Nasional. P. 206-214.

Chen, S., B. Fan, B. Liu, M. Yu, S. Zhao, M. Zhu, T. Xiong and K. Li. 2006. Genetic variations of 13 indigenous Chinese goat breed based on Cytochrome $\mathrm{b}$ gene sequence. Biochem. Genet. 44:89-99.

Doadrio, I. and A. Perdices. 2005. Phylogenetic relationships among the Ibero-African cobitids (Cobitis, Cobitidae) based on Cytochrome b sequence data. Mol. Phylogenet. Evol. 37:484-493.

GenBank. www.ncbi.nlm.nih.gov (Accessed on $25^{\text {th }}$ August 2016)

Guang-Xin, E., Y. F. Huang, N. Liu, Y. Zhao, J. N. He, R. S. Na, Z. Q. Zhao, C. D. Jiang, J. H. Zhang, Y. H. Ma, L. P. Chen, X. Y. Qiu, Y. W. Sun, Y. Z., Y. Z. Sun, C. H. Yu and S. Y. Wei. 2015. Characteristics of the mitochondrial genome of four native goats in China (Capra hircus). Mitochondrial DNA, Early Online: 1-2

Farias, I. P., G. Orti, I. Sampaio, H. Schneider and A. Meyer. 2001. The Cytochrome b gene as a phylogenetic marker: the limits of resolution for analyzing relationships among Cichlid fishes. J. Mol. Evol. 53:89-103.

Hassanin, A., C. Bonillo, B. X. Nguyen and C. Cruaud. 2010. Comparisons between mitochondrial genomes of domestic goat (Capra hircus) reveal the presence of numts and multiple sequencing errors. Mitocondrial DNA. 21 (4):68-76.

Jiyanto, E. Kurnianto and Sutopo. 2014. The genetic diversity of Kejobong goat based on Cytochrome $\mathrm{b}$ gene. J. Indonesian Trop. Anim. Agric. 39(2):75-82.

Karsinah, Sudarsono, L. Setyobudi and $H$. Aswidinoor. 2002. Keragaman genetik plasma nutfah jeruk berdasarkan analisis penanda RAPD. J. Biotek. Pertanian. 7(1): 8-16.

Kocher, T. D., W. K. Thomas, A. Meyer, S. V. Edwards, S. Paabo, F. X. Villablanca and A. C. Wilson. 1989. Dynamics of mitochondrial DNA evolution in animals: Amplification and sequencing with conserved primer. Proc. Natl. Acad. Sci.
USA. 86 (16):6196-6200.

Kul, B. C. and O. Ertugrul. 2011. MtDNA diversity and phylogeography of some Turkish native goat breeds. Ankara Univ. Vet. Fak. Derg. 58 : 129-134.

Liu, Y. P., S. X. Cao, S. Y. Chen, Y. G. Yao and T. Z. Liu. 2009. Genetic diversity of Chinese domestic goat based on the mitochondrial DNA sequence variation. J. Anim. Breed. Genet. 126:80-89.

Machugh, D. E. and D. G. Bradley. 2001. Livestock genetic origins: goat buck the trend. Proc. Natl. Acad. Sci. USA. 98(10): 5382-5384.

Manceau, V., J. P. Crampe, P. Boursot and P. Taberlet. 1999. Identification of evolutionary significant unit in the spanish wild goat, Capra pyrenaica (Mammalia artiodactyla). J. Anim. Consv. 2: 33-39.

Mason, I. L. 1984. Evolution of Domesticated Animals. Prentice Hall Press, New York, USA. P. 468.

Ministry of Agriculture. 2016. Statistic Data of Animal Husbandry 2015. Directorate General of Animal Husbandry and Veterinary. Ministry of Agriculture, Republic of Indonesia.

Naderi, S., H. R. Rezaeia, F. Pompanona, M. G. B. Blumd, R. Negrinie, H. R. Naghasha, O. Balkızf, M. Mashkourg, O. E. Gaggiottia, P. A. Marsane, A. Kencef, J. D. Vigneg, and P. Taberlet. 2008. The goat domestication process inferred from large-scale mitochondrial DNA analysis of wild and domestic individuals. PNAS. 105(46): 17659-17664

Pakpahan, S., W. T. Artama, R. Widayanti and I. G. Suparta. 2016a. Genetic characteristics and relationship in different goat population of Indonesia based on cytochrome $b$ gene sequences. Asian J. Anim. Sci. 10(1):29-38.

Pakpahan, S., W. T. Artama, R. Widayanti and I. G. Suparta. 2016b. Molecular phylogenetic of Hutan Sumatera goat (Sumatran serow) and domestic goat (Capra hircus) in Indonesia based on analysis mitochondrial Cytochrome $b$ gene. Asian J. Anim. Vet. Adv. 11(6):331-340.

Pidancier, N., S. Jordan, G. Luikart and P. Taberlet. 2006. Evolutionary history of the genus Capra (Mammalia, Artiodactyla): discordance between mitochondrial DNA and Y-chromosome phylogenesis. Mol. Phyl. Evol. 40:739-749. 
Pietro, P., F. Maria, G. Gianfranco and E. Giuseppe. 2003. The complete nucleotide sequence of goat (Capra hircus) mitochondrial genome. DNA Seq. 14(3): 199-203.

Poerba, Y. S. dan Yuzammi. 2008. Pendugaan keragaman genetik Amorphophallus titanium Becc. berdasarkan marka Random Amplified Polymorphic DNA. Biodiversitas 9(2):103-107.

Prawirodigdo, S., T. Herawati and B. Utomo. 2003. Penampilan Peternakan Kambing dan Potensi Bahan Pakan Lokal sebagai Komponen Pendukungnya di Wilayah Propinsi Jawa Tengah. Proceedings, Lokakarya Nasional Kambing Potong, Balai Pengkajian Teknologi Pertanian Jawa Tengah. P. 157-164.

Sambrook, J., E. F. Fritsch and T. Maniatis. 1989. Molecular Cloning. A Laboratory Manual. Cold Spring Harbour Laboratory Pr. NewYork.

Santoso, T. J., D. W. Utami and E. M. Septiningsih. 2006. Analisis sidik jari plasma nutfah kedelai menggunakan marka SSR. J. Agro Biogen. 2 (1): 1-7.

Shackleton, D. M. 1997. Wild Sheep and Goats and their Relatives: Status Survey and Conservation Action Plan for Caprinae. IUCN, Gland, Switzerland.
Slechtová, V., J. Bohlen, J. Freyhof and P. Ráb. 2006. Moleculer phylogeny of the southeast freshwater fish family Botiidae (Teleostei: Cobitoidea) and the origin of polyploidy in their evolution. Mol. Phylogenet. Evol. 39:529-541.

Sodiq, A. and Z. Abidin. 2008. Meningkatkan Produksi Susu Kambing Peranakan Ettawa. Agromedia Pustaka. Jakarta.

Takada, T., Y. Kikkawa, H. Yonekawa, S. Kawakami and T. Amano. 1997. Bezoa (Capra argagrus) is a material candidate for ancestor of domestic goat (Capra hircus): evidence from the mitochondrial DNA diversity. Biochem. Genet. 35 (9): 315-326.

Tamura, K., G. Stecher, D. Peterson, A. Filipski and S. Kumar. 2013. MEGA6: Molecular evolutionary genetics analysis version 6.0. J. Mol. Biol. Evol.30:2725-2729.

Thompson, J.D., D. G. Higgins and T.J. Gibson. 1994. CLUSTAL W: Improving the sensitivity of progressive multiple sequence alignment through sequence weighting, Position specific gap penalties and weight matrixchoice. Nucleic Acid Res. 22:46734680.

Widayanti, R., D. D. Solihin, D. Sajuthi and R. R. D. Perwitasari. 2006. Kajian penanda genetik gen Cytochrome b pada Tarsius $s p$. J. Sain. Vet. 24(1):126-132. 